\title{
Czochralski Method of Crystal Growth in the Scientific Literature: An Informetric Study
}

\author{
K. SANGWAL* \\ Department of Applied Physics, Lublin University of Technology \\ Nadbystrzycka 38, 20-618 Lublin, Poland
}

\begin{abstract}
Bibliometric data on the growth dynamics of papers devoted to the Czochralski method of crystal growth and citations to these papers published in the scientific literature are analyzed using mathematical functions based on progressive nucleation mechanism and power-law approaches. It is shown that the basic concepts of progressive nucleation mechanism originally developed for crystal growth can satisfactorily be applied to explain the citation behavior of papers published on the Czochralski method of crystal growth. It is also found that: (1) cumulative number $N(t)$ of papers and cumulative number $L(t)$ of citations to them at time $t$ are mutually related, and (2) as determined by the plot of the parameter $L(t) / t^{2}$ against the number $\Delta N(t)$ of papers published per year, the citation behavior of papers published on the Czochralski method follows three distinct periods: 1954-1988, 19882001 and 2001-2012, which are related to the dependence of the number $\Delta N(t)$ of papers published per year on publication time $t$.
\end{abstract}

DOI: $10.12693 /$ APhysPolA.124.173

PACS: 81.10.Aj, 89.20.-a, 89.90.+n

\section{Introduction}

Early fifties of the 20th century witnessed the publication of three scientific contributions: (1) a paper entitled "Growth of germanium single crystals" by Teal and Little [1], (2) a paper entitled "The growth of crystals and the equilibrium structure of their surfaces" by Burton, Cabrera and Frank [2], and (3) a monograph entitled "Crystal Growth" by Buckley [3]. These contributions provided enormous impetus to research activities in the field of crystal growth and served as percursors for the nucleation and development of various subfields of crystal growth. For example, in later years the paper by Teal and Little [1] on the growth of germanium became a reference point for the growth of a variety of materials from the melt by the method introduced by Jan Czochralski, and the paper by Burton et al. [2] became a central point for the investigation of the kinetics and mechanism of growth of crystals from different phases and the growth morphology of crystals. For a long time Buckley's monograph [3] also remained a valuable source of literature on crystal growth and a source of inspiration in the investigations of the effect of impurities on the growth morphology of crystals from solutions. In this monograph the method used by Czochralski was called "crystal pulling". However, since early nineteen fifties it is usually referred to as "Czochralski method" of crystal growth although this name of the method (i.e. Czochralski method) has been used before in 1921 as noted by Wilke and Bohm [4].

Jan Czochralski was a self-made man. Formally, he did not have University education but thanks to hard work,

*e-mail: k.sangwal@pollub.pl determination and vision, he became a renowned metallurgist in Germany and during 1924-1929 was president of the German Society for Metals Science. Later, President of Poland Ignacy Mościcki, inter alia, persuaded him to return to Poland. In 1928 he was appointed professor of metallurgy and metals science in Warsaw University of Technology, which also conferred on him the title of doctor honoris causa in 1929. The outbreak of the Second World War interrupted his diverse scientific activities. After the war the new communist regime blamed him of collaboration with the Nazis and in line with the political propaganda the Warsaw University of Technology stripped him of his job. Thereafter he returned to his native Kcynia where he died in 1953. In the article entitled: "Professor Jan Czochralski (1885-1953) and his contribution to the art and science of crystal growth", Tomaszewski [5] briefly describes the life and achievements of Jan Czochralski in chronological order. Recently, Tomaszewski published a documented biography of his life and achievements [6].

In Poland Czochralski's name remained condemned during the communist regime but, thanks to his method, his name steadily grew in fame with the continuously increasing number of scientific papers devoted to the growth and characterization of crystals of various materials. This caused the appearance of publications in nineteen eighties praising the contribution of Czochralski as a Pole [7], but the Polish academic community remained unconcerned with his after-war fate. After the transformation of the political system in 1989, it occurred to the present author during his stay in Spain in 1990 to organize a crystal growth society in Poland. The concept of founding the society in Poland originated from crystal growth societies, with not large number of members, functioning in Spain and Italy and organiz- 
ing crystal growth meetings at national levels. In May 1991 the present author organized in Częstochowa, where he was then working, the first inaugural meeting/conference of Polish Society for Crystal Growth with about 20 active crystal growers from the country. It was in this Conference that the first, inaugural lecture, conceived as Czochralski Lecture, was initiated, despite the accepted condemnation in academic circles stretching since 1945 , in recognition of the scientific contribution of Professor Jan Czochralski as a great Polish Scientist. This Czochralski Lecture is now a traditional feature of every Polish Conference on Crystal Growth.

Since its foundation in 1991 Polish Society for Crystal Growth, with immense involvement, among others, of Professor Anna Pajaczkowska, had been making every effort to restore the good name of Professor Jan Czochralski. Two recent events, one at the academic level and the other at the national level, are very important steps in this direction. In June 2011 the Senate of the Warsaw University of Technology adopted a resolution to restore the dignity of Professor Jan Czochralski and the place he deserves in the academic community. On December 7, 2012 Polish Parliament adopted a resolution declaring 2013 as Year of Czochralski. These two resolutions are marks of tributes to Czochralski as a patriot. The present issue of Acta Physica Polonica A is dedicated to commemorate this Year of Czochralski.

The present author has the honor of being invited by the Guest Editors of the planned special issue of Acta Phys. Pol. A to contribute a paper on Czochralski method. Since 1968 the author has been associated with crystal growth but regretfully he never grew crystals by the Czochralski method. Therefore, when the author received the invitation, he had the choice of refusing it and thereby not contributing anything to the special issue. However, in view of the author's fascination for Professor Czochralski as a scientist, the author decided to accept the invitation and pay a tribute to him by contributing a paper on the growth of the scientific literature bearing his imprint.

The number of scientific papers devoted to growth and characterization of crystals of various materials by the Czochralski method and their citations has grown steadily since the publication of the paper by Teal and Little [1]. In recent years about 400 papers based on Czochralski method are published every year, which receive about 5500 citations every year (see below). These numbers are huge in comparison with less than 10 papers published by an individual mature author, working in a research team, per year and less than 200-300 citations to his/her papers per year [8,9]. Citation analysis is an active field of research in informetrics. Therefore, it is of interest to carry out an informetric study of papers related to Czochralski method and citations to these papers.

It is well known that the cumulative number of items such as journals, articles and authors in different scientific fields initially increases with time and then after a particular period it attains a saturation value. If one considers the growth of items per year (i.e. their number in successive years), one observes that this growth occurs at a relatively slow rate initially, followed by an exponential increase, and, in some cases, finally the growth declines after a certain time, giving rise to a sigmoidal shaped (S-shaped) curve. Among the different equations of various models to analyze the above type of growth behavior of items, power-law, exponential and logistic functions are commonly used [10-15]. Recently, Sangwal $[8,9,16]$ proposed a new equation, based on progressive nucleation mechanism (PNM) of a solid phase during its crystallization in a closed liquid system of fixed volume.

The aim of this paper is to analyze the bibliometric data on the growth dynamics of papers devoted to the Czochralski method of crystal growth and citations to these papers published in the scientific literature using mathematic functions based on progressive nucleation mechanism and power-law approaches. The structure of the paper is as follows: the basic concepts of progressive nucleation mechanism and the basic equations as applied to citation analysis are described in Sects. 2 and 3, respectively, the appropriate bibliometric data for the analysis are presented in Sect. 4, whereas the time dependence of the growth of papers and their citations and the relationship between the number of citations and the number of papers fetching them are discussed in Sects. 5 and 6, respectively. Finally, the main conclusions drawn from the study are given in Sect. 7 .

\section{Basic concepts of progressive nucleation mechanism}

In overall crystallization of a solid phase occurring in a closed liquid system of fixed volume it is assumed [17] that nucleation of the solid phase occurs on a total number $N_{\mathrm{a}}$ of active centers present in the crystallizing medium and each nucleus grows independently of the other nucleating and growing crystallites. The number of active centers is exhausted progressively during the nucleation process, thereby determining the time dependence of nucleation on active centers and finally the overall crystallization of the solid phase resulting from the growth of the nuclei by the attachment of growth units occurs in the liquid phase. This type of growth is known as progressive nucleation mechanism. The theoretical aspects of progressive nucleation mechanism are well developed in the field of crystallization [17].

The time dependence of cumulative number of items such as journals, articles and authors in a scientific field is also a continuous process in which new items are produced progressively. An author publishing $N$ papers in his research career and a research paper published by a given author receiving a total of $L$ citations are typical examples of closed systems. In these examples, the author publishing papers and the paper receiving citations are sources producing items (i.e. papers and citations, respectively). These source-items isolated systems are 
simple in nature because the source is an individual entity. However, when a group of sources produces items, one deals with complex systems.

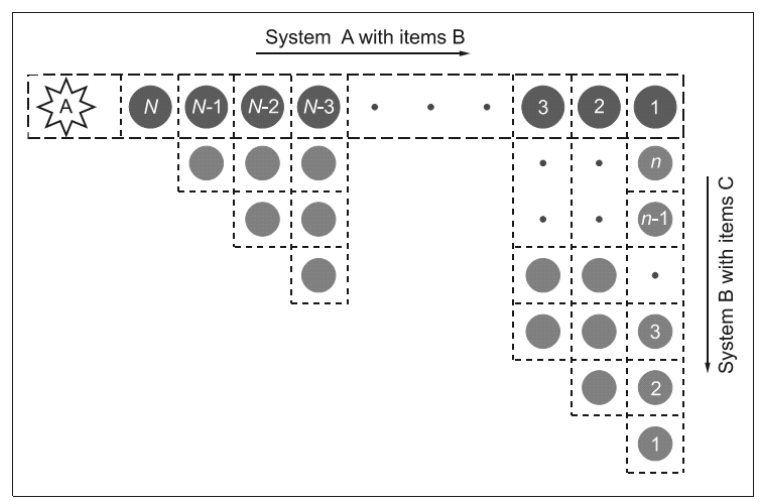

Fig. 1. Schematic illustration of process of generation of primary items B by a hypothetical source A (system 1 ), and generation of secondary items $\mathrm{C}$ by the primary item B behaving as secondary source (system 2). System 1 is composed of individual source A and B items of an array of relatively dark circles enclosed in the horizontal rectangle. Systems 2 are composed of individual secondary sources B (i.e. previous items B) and new items $\mathrm{C}$ in the form of different entities shown by arrays of grey circles in columns running downwards from the horizontal array of secondary sources B.

The process of generation of items B by an individual source A is shown schematically in Fig. 1. These items are indicated by an array of relatively dark, large circles enclosed by the horizontal rectangle. This individual source A and the array of items B is said to compose system A, which is essentially a primary or simple system. Now let us suppose that each of the items B produced by the source $\mathrm{A}$ also behaves as an independent source of generation of new or secondary items as shown schematically by parallel arrays of grey, relatively small, circles in columns running downwards from the horizontal array of items. This new, independent source, say 1 , in the array of items B and its secondary items $C$ form system B. Note that we distinguish system B from system A because it is composed of a secondary source B and secondary items $\mathrm{C}$ different from the source $\mathrm{A}$ and items $\mathrm{B}$ of system A. However, each secondary source B and the secondary items generated by it also form an independent system similar to system A. Thus, our entire system is composed of one primary source A, an array of primary items B behaving subsequently as secondary sources generated by the primary source $\mathrm{A}$, and items $\mathrm{C}$ generated by each of the secondary sources B (i.e. primary items) which produce items $\mathrm{C}$ independent of each other. This is an example of complex systems represented by the outer closed frame.

The above example of the process of generation of secondary items $\mathrm{C}$ by secondary sources $\mathrm{B}$, which are essentially primary items B produced successively by primary source A also applies to the process of citations of suc- cessively published papers by a hypothetical author A. Then the horizontal array of dark circles represents papers B, starting from Paper 1, published successively by the hypothetical author A, whereas the parallel arrays of grey circles arranged in columns running downwards denote citations $\mathrm{C}$. If one denotes the number of cumulative papers published by an author at time $t$ by $N(t)$ and the number of citations received by the $i$-th paper at time $t$ by $L_{i}(t)$, the cumulative citations $L(t)$ received by cumulative papers $N(t)$ successively published papers after time $t$ by the author may be considered as an example of complex systems.

\section{Basic equations for citation data}

The author recently applied the above PNM approach to describe the growth dynamics of citations of individual authors $[8,9]$, cumulative citations of individual papers of individual authors [16], articles in three randomly selected databases from 20 different databases in humanities, social sciences, and science and technology [18] and the growth of journals, articles and authors in malaria research [18], and to analyze and compare the scientific activities of different authors [8, 19]. The basic equations of the PNM approach used in this paper for the analysis of the bibliometric data related to citations to the Czochralski method of crystal growth are given below.

The fraction $\alpha(t)$ of cumulative items $y(t)$ at time $t$ generated by an individual source may be given by $[8,9]$ :

$$
\alpha(t)=\frac{y(t)}{y_{0}}=\left[1-\exp \left(-\left(\frac{t}{\Theta}\right)^{q}\right)\right],
$$

where $y_{0}$ is the maximum number of items that the source can give, the time constant

$$
\Theta=\frac{q^{1 / q}}{\kappa J_{\mathrm{s}}}
$$

and the exponent

$$
q=1+\nu d
$$

In the above equations, $J_{\mathrm{s}}$ is the rate of stationary nucleation of items and $\kappa$ is the shape factor (e.g. $\kappa=16 \pi / 3$ for a sphere) and the time

$$
t=Y-Y_{0},
$$

where $Y$ is the year of the items $y(t)$ and $Y_{0}$ is the actual or extrapolated year when $\alpha(t)=0$. In Eq. (3) the parameter $d$ denotes the dimensionality of nuclei of items, which is equal to 1, 2 and 3 for one-, two- and three-dimensions, respectively, whereas the exponent $\nu=1 / 2$ and 1 for the growth of the nuclei controlled by volume diffusion and mass transfer, respectively.

The cumulative fraction $\alpha_{\text {sum }}(t)$ of items generated by successively appearing sources at time $t$ by a system or set of sources characterized by different values of time constant $\Theta$ and exponent $q$ in Eq. (1) as a function of their generation duration $t$ may be expressed by a similar relation $[9,19]$, i.e.

$$
\alpha_{\text {sum }}(t)=\alpha_{\text {sum } 0}\left[1-\exp \left(-\left(\frac{t}{\Theta_{0}}\right)^{q_{0}}\right)\right] \text {, }
$$

where $\alpha_{\text {sum0 }}$ is the sum of all of the maximum fractions 
of items from the collective of sources, and $\Theta_{0}$ and $q_{0}$ are new time constant and exponent, respectively, describing the resultant behavior of the entire collective of sources. Since the ratio $\alpha_{\text {sum }} / \alpha_{\text {sum0 }}$ of items may be considered as an average cumulative fraction of items and is related to their cumulative number $y_{\text {sum }}$, Eq. (5) may be written as

$$
\frac{\alpha_{\text {sum }}(t)}{\alpha_{\text {sum } 0}}=\frac{y_{\text {sum }}}{y_{\text {sum } 0}},
$$

where $y_{\text {sum0 }}$ is the maximum number of items that the system can attain. Then Eq. (5) takes the form

$$
y_{\text {sum }}(t)=y_{\text {sum } 0}\left[1-\exp \left(-\left(\frac{t}{\Theta_{0}}\right)^{q_{0}}\right)\right] \text {, }
$$

where $y_{\text {sum0 }}$ is the sum of the maximum numbers of items generated by the collective of sources. Differentiation of Eq. (7) with respect to $t$ gives the change in the number $y_{\text {sum }}$ of items with time $t$ (i.e. the number $\Delta y_{\text {sum }}$ of items produced by the source per unit time) in the form

$$
\Delta y_{\mathrm{sum}}=\frac{\mathrm{d} y_{\mathrm{sum}}(t)}{\mathrm{d} t}=\frac{y_{\mathrm{sum} 0} q_{0}}{\Theta_{0}^{q_{0}}} t^{q_{0}-1} \exp \left(-\left(\frac{t}{\Theta_{0}}\right)^{q_{0}}\right) .
$$

According to this equation the change in the number $\Delta y_{\text {sum }}(t)$ of items initially increases and then decreases with an increase in time $t$ after passing through a maximum value of $\Delta y_{\text {sum }}(t)$ at a particular value of $t_{\max }$. In the increasing part of the number $\Delta y_{\text {sum }}(t)$ of items produced per unit time by the successively appearing sources, the time dependence of $\Delta y_{\text {sum }}(t)$ can also be described by the empirical relation

$$
\Delta y_{\text {sum }}=\lambda_{0}\left[1-\exp \left(-\left(\frac{t}{\Theta_{\text {sum }}}\right)^{q_{\text {sum }}}\right)\right],
$$

where $\Theta_{\text {sum }}$ and $q_{\text {sum }}$ are new time constant and exponent, approximately equal to $\Theta_{0}$ and $q_{0}$, respectively, and the constant $\lambda_{0}=y_{\mathrm{sum} 0} / z$, with the parameter $z$ is related to the total number of sources producing cumulative items $y_{\text {sum }}(t)$
It should be mentioned that, when the approximation $\mathrm{e}^{x}=1+x$ holds, both Eqs. (1) and (5) transform to the power-law relation

$$
y(t)=\left(\frac{y_{0}}{\theta^{p}}\right) t^{p},
$$

where the $\theta$ denotes the time constants $\Theta$ and $\Theta_{0}$, and $p$ denotes the exponents $q$ and $q_{0}$ of Eqs. (1) and (5), respectively. Note that Eq. (5) is derived on the assumption that individual sources are produced successively at equal intervals of time. In the case of similar individual sources B characterized by the same time constant $\Theta$ and the same exponent $q$, this means that $p=q$ in Eq. (10).

Equations (7)-(10) can be applied to describe the citation behavior of papers published by an individual author or by different authors on a particular topic such as the Czochralski method of crystal growth. In that case we replace cumulative items $y_{\text {sum }}$ by cumulative number $L$ of citations, the maximum number of items $y_{\text {sumo } 0}$ by the sum of the maximum number $C_{0}$ of citations from the collective of successively published $N$ papers and the number $\Delta y$ of items generated per unit time by the number $\Delta N$ of papers published per year and by the number $\Delta L$ of citations received by the papers per year.

\section{Bibliometric data for analysis}

We used Thomson Reuters' ISI Web of Knowledge database to collect the basic bibliometric data under the topic "Czochralski". The basic data involving the cumulative number $N(Y)$ of papers and the cumulative number $L(Y)$ of citations received by the papers in the year $Y$, and the number of papers $\Delta N(Y)$ published per year and the number $\Delta L(Y)$ of citations per year in the year $Y$. Here $\Delta N(Y)$ and $\Delta L(Y)$ are the increments in the values of $N(Y)$ of papers and $L(Y)$ of citations of the papers in the year $Y$ are given in Table I. The data were collected during 21-22 January 2013.

Bibliometric data on papers dealing with the Czochralski method and their citations.

TABLE I

\begin{tabular}{c|c|c|c|c|c|c|c|c|c|c|c|c|c|c}
\hline \hline Year & $\Delta N$ & $N$ & $\Delta L$ & $L$ & Year & $\Delta N$ & $N$ & $\Delta L$ & $L$ & Year & $\Delta N$ & $N$ & $\Delta L$ & $L$ \\
\hline 1954 & 1 & 1 & 0 & 0 & 1974 & 27 & 178 & 159 & 870 & 1994 & 266 & 1970 & 1251 & 12126 \\
1955 & 0 & 1 & 0 & 0 & 1975 & 18 & 196 & 108 & 978 & 1995 & 286 & 2254 & 1685 & 13811 \\
1956 & 0 & 1 & 0 & 0 & 1976 & 25 & 221 & 131 & 1109 & 1996 & 302 & 2556 & 1817 & 15628 \\
1957 & 1 & 2 & 2 & 2 & 1977 & 37 & 258 & 175 & 1284 & 1997 & 398 & 2954 & 2408 & 18036 \\
1958 & 1 & 3 & 1 & 3 & 1978 & 31 & 289 & 166 & 1450 & 1998 & 344 & 3298 & 2131 & 20167 \\
1959 & 0 & 3 & 4 & 7 & 1979 & 32 & 321 & 136 & 1586 & 1999 & 405 & 3703 & 3311 & 23478 \\
1960 & 2 & 5 & 4 & 11 & 1980 & 49 & 370 & 233 & 1819 & 2000 & 385 & 4088 & 3435 & 26913 \\
1961 & 3 & 8 & 9 & 20 & 1981 & 55 & 425 & 393 & 2212 & 2001 & 374 & 4462 & 3365 & 30278 \\
1962 & 4 & 12 & 9 & 29 & 1982 & 53 & 478 & 400 & 2612 & 2002 & 422 & 4884 & 4075 & 34353 \\
1963 & 5 & 17 & 21 & 50 & 1983 & 60 & 538 & 466 & 3078 & 2003 & 419 & 5303 & 3955 & 38308 \\
1964 & 5 & 22 & 24 & 74 & 1984 & 48 & 586 & 488 & 3566 & 2004 & 434 & 5737 & 4607 & 42915 \\
1965 & 9 & 31 & 62 & 136 & 1985 & 65 & 651 & 491 & 4057 & 2005 & 438 & 6175 & 4800 & 47715 \\
1966 & 12 & 43 & 51 & 187 & 1986 & 73 & 724 & 599 & 4656 & 2006 & 425 & 6600 & 4545 & 52260 \\
1967 & 15 & 58 & 64 & 251 & 1987 & 78 & 802 & 675 & 5331 & 2007 & 413 & 7013 & 5028 & 57288 \\
1968 & 14 & 72 & 53 & 304 & 1988 & 55 & 857 & 873 & 6204 & 2008 & 359 & 7372 & 4943 & 62231 \\
1969 & 11 & 83 & 51 & 355 & 1989 & 73 & 930 & 913 & 7117 & 2009 & 330 & 7702 & 4947 & 67178 \\
1970 & 7 & 90 & 54 & 409 & 1990 & 94 & 1024 & 710 & 7827 & 2010 & 350 & 8052 & 5315 & 72493 \\
1971 & 19 & 109 & 71 & 479 & 1991 & 207 & 1231 & 893 & 8720 & 2011 & 352 & 8404 & 5848 & 78341 \\
1972 & 23 & 132 & 101 & 580 & 1992 & 191 & 1422 & 745 & 9465 & 2012 & 311 & 8715 & 5554 & 83895 \\
1973 & 19 & 151 & 132 & 712 & 1993 & 282 & 1704 & 1410 & 10875 & & & & &
\end{tabular}


Values of best-fit parameters of Eq. (7) for $N(t)$ and $L(t)$ data.

TABLE II

\begin{tabular}{c|c|c|c|c|c}
\hline \hline Data & Range & $Y_{0}$ & $N_{0}$ (papers) & $\Theta$ or $\Theta_{0}$ or $C_{0}$ [cites] & $q$ or $q_{0}$ [years] \\
\hline$N(Y)$ & all & 1954 & $10254 \pm 268$ & $51.84 \pm 0.45$ & $5.758 \pm 0.108$ \\
& $<1995$ & 1954 & $5985 \pm 5637$ & $59.13 \pm 20.09$ & $4.911 \pm 0.112$ \\
& & 1960 & $10592 \pm 368$ & $46.39 \pm 0.61$ & $4.911 \pm 0.112$ \\
& $>1980$ & 1980 & $18541 \pm 5728$ & $39.42 \pm 8.67$ & $2.041 \pm 0.135$ \\
$L(Y)$ & all & 1954 & $183306 \pm 19481$ & $63.04 \pm 1.66$ & $5.767 \pm 0.112$
\end{tabular}

Values of best-fit parameters of power-law relation (10).

TABLE III

\begin{tabular}{c|c|c|c}
\hline \hline Data & $Y_{0}$ [year] & $y_{0} / \Theta^{p}$ & $p$ \\
\hline$N(Y)$ & 1954 & $(1.4 \pm 0.3) \times 10^{-4}\left[\right.$ papers $/$ year $\left.^{p}\right]$ & $4.988 \pm 0.047$ \\
$L(Y)$ & 1954 & $(1.2 \pm 0.3) \times 10^{-3}\left[\right.$ cites $/$ year $\left.^{p}\right]$ & $3.908 \pm 0.082$
\end{tabular}

The publication period $t=Y-Y_{0}$, where $Y_{0}$ is the year of publication of the first paper and $Y$ is the year of publication under consideration. The data were analyzed using Origin software (version 4.1). The procedure following the analysis of the data is described elsewhere $[8,9]$. In cases when it was difficult to establish best fits, two different sets of the values of the constants were recorded. Examples of this type of sets of the constants are the $N(t)$ data given in Table II.

\section{Growth of papers published on Czochralski method and their citations with time}

In this section the growth dynamics of papers devoted to the Czochralski method of crystal growth and citations to these papers published in the scientific literature are analyzed using the theoretical equations presented above.

Figure 2 shows the plots of cumulative number $N(t)$ of papers devoted to the Czochralski method and their cumulative number $L(t)$ of citations on their publication year $Y$. Solid and dashed lines in the figure are drawn according to PNM relation (7) and power-law relation (10), respectively, with the best-fit parameters listed in Tables II and III. It may be seen that the $N(t)$ data are better described by PNM relation alone but the $L(t)$ data can be described satisfactorily by both relations. In the former case, significant deviations are observed for data after 1985. In particular, the tendency of retardation in the values of $N(t)$ with $t$ after 2005 is not reproduced by the power-law relation. Moreover, the values of $p$ obtained by power-law relation (10) are somewhat lower than those obtained by the PNM relation.

Figure 3 presents the plots of $\Delta N(t)$ papers per year devoted to the Czochralski method and $\Delta L(t)$ citations per year on their publication year $Y$. The solid lines are plotted according to Eq. (8) with the best-fit values of the parameters listed in Table II for data in the entire range. As seen from these plots, except for noticeable deviations in a narrow range of publication of the $\Delta N(t)$ data from the theoretically predicted curve during $1984-$ 1991, Eq. (8) satisfactorily reproduces the data.
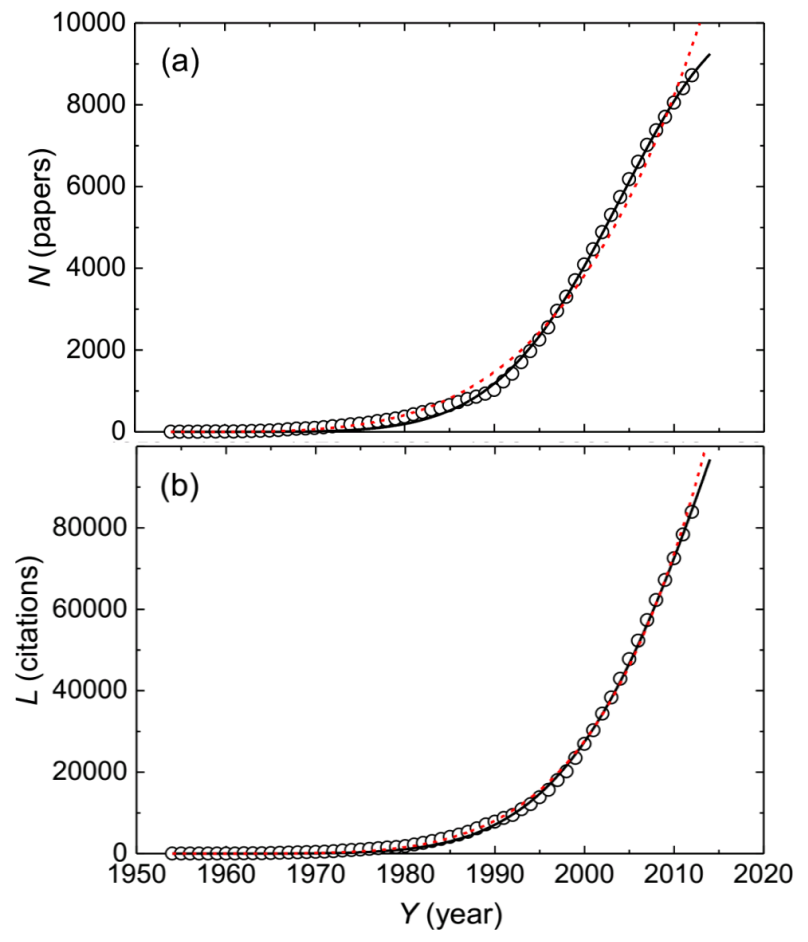

Fig. 2. Dependence of (a) cumulative papers $N(t)$ devoted to the Czochralski method and (b) their cumulative citations $L(t)$ on their publication year $Y$. Solid and dashed lines are plotted according to best-fit parameters, listed in Tables II and III, of PNM and power-law relations, respectively.

Upon taking logarithm on both sides of power-law relation (10) one obtains

$$
\ln y(t)=\ln \left(\frac{y_{0}}{\theta^{p}}\right)+p \ln t
$$

which predicts a linear dependence of $\ln y(t)$ on $\ln t$ with slope $p$ and intercept $A=\ln \left(y_{0} / \theta^{p}\right)$. Figure 4 shows the dependence of $\ln N(t)$ and $\ln L(t)$ on $\ln \left(Y-Y_{0}\right)$. Linear plots in the figure are drawn with the best-fit values of constants $\mathrm{A}$ and $p$ for $\ln \left(Y-Y_{0}\right)<3.7$ given in Table IV. 


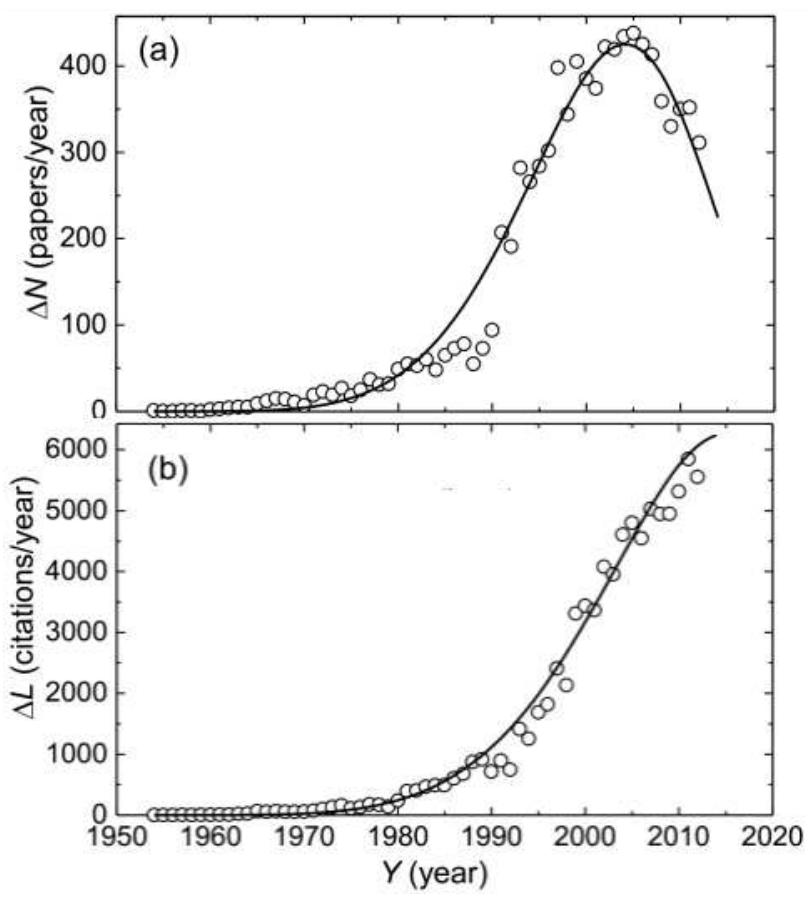

Fig. 3. Dependence of (a) papers $\Delta N(t)$ per year devoted to the Czochralski method and (b) citations $\Delta L(t)$ per year on their publication year $Y$. Solid lines are plotted according to best-fit parameters, listed in Table II of PNM relation.

It may be noted that the plots of $\ln L$ against $\ln t$ may be described by the linear relation practically in the en-

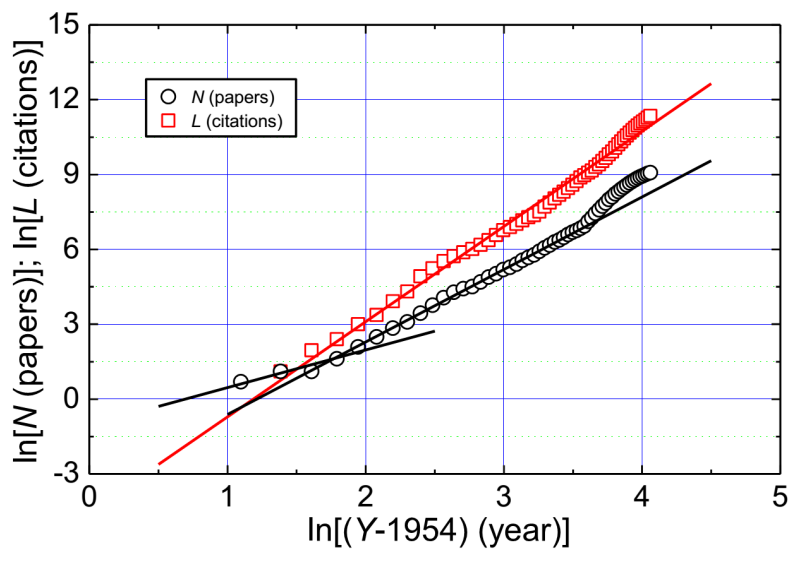

Fig. 4. Dependence of $\ln N(t)$ and $\ln L(t)$ devoted to the Czochralski method on $\ln \left(Y-Y_{0}\right)$. Linear plots are drawn according to best-fit parameters listed in Table III. See text for details.

tire time range with the values of $y_{0} / \theta^{p}$ and $p$ very similar to those obtained by fitting the $L(t)$ data directly by Eq. (10); see Table III. In contrast to the $L(t)$ data, the data of $\ln N$ as a function of $\ln t$ can only be represented by the linear dependence in different time intervals and the value of the exponent $p$ is lower than that given in Table III. However, a careful examination of the two plots shows that, except for the first two points below $\ln \left(Y-Y_{0}\right)<1.5$ for $N(t)$ data, the trends of the plots of the two data are very similar, suggesting that $N(t)$ and $L(t)$ are mutually related.

Values of best-fit parameters of linear relation (13).

TABLE IV

\begin{tabular}{c|c|c|c|c|c}
\hline \hline Data & $\ln t$ range & $Y_{0}$ [year] & $-A$ & $p$ & $\mathrm{RC}$ \\
\hline$N(Y)$ & $1.5-3.5$ & 1954 & $3.520 \pm 0.078$ & $2.906 \pm 0.026$ & 0.9990 \\
& $<2$ & 1954 & $1.052 \pm 0.492$ & $1.511 \pm 0.309$ & 0.9427 \\
$L(Y)$ & all & 1954 & $4.523 \pm 0.160$ & $3.814 \pm 0.048$ & 0.9958
\end{tabular}

Values of best-fit parameters of Eq. (14).

TABLE V

\begin{tabular}{c|c|c|c|c}
\hline \hline$L_{\text {sum }}(t) / t^{2}$ range [papers $/$ year $\left.^{2}\right]$ & $Y$ range [years] & $a$ [citations $/$ year $^{2}$ ] & $b\left[\right.$ year $^{-1}$ ] & RC \\
\hline$<5$ & $1954<Y<1987$ & $0.436 \pm 0.119$ & $0.0616 \pm 0.0031$ & 0.9605 \\
$5<L / t^{2}<12$ & $1987<Y<2001$ & $3.503 \pm 0.784$ & $0.0194 \pm 0.0028$ & 0.8851 \\
$>17$ & $2005<Y<2012$ & $40.29 \pm 3.02$ & $-0.0503 \pm 0.0079$ & 0.9233
\end{tabular}

\section{Relationship between papers published and citations received by them}

Equations (7), (8) and (10) are derived on the assumption that the change in the number $N$ of papers (i.e. individual sources) per unit time, defined by $\Delta N$, occurs successively and is constant. In other words, the cumulative number $N$ of papers published by an author increases linearly with time $t$ during his/her publication career (see above). In reality, this assumption is frequently violated. In order to explain these violations we consider Eq. (7) as applied for the time dependence of citations according to the progressive nucleation mechanism.

In the time interval $0<t<\Theta_{0}$ when the approximation $\mathrm{e}^{x}=1+x$ is valid, the cumulative citations $L(t)$ of cumulative $N$ papers in the entire publication career lim- 
ited to time $T$ such that $\Theta_{0}<T / 3$ may be given by [19]

$$
L(t)=\lambda_{0} \frac{\Delta N}{\Theta_{0}^{q_{0}-1}} t^{q_{0}},
$$

where the cumulative papers $N=\Delta N \Theta_{0}$ and $\lambda_{0}$ is a citability parameter related to the $L(t) / \alpha_{\text {av }}(t)=$ $L\left(\Theta_{0}\right) / \alpha_{\text {av }}\left(\Theta_{0}\right)$. Since $q_{0} \approx 2$, Eq. (12) may also be rearranged in the form

$$
\ln L(t)=\ln \left(\frac{\lambda_{0}}{\Theta_{0}} t\right)+\ln N(t) .
$$

Equation (13) predicts a linear dependence of $\ln L(t)$ on $\ln N(t)$ with slope equal to unity and intercept equal to $\ln \left(\lambda_{0} t / \Theta_{0}\right)$.

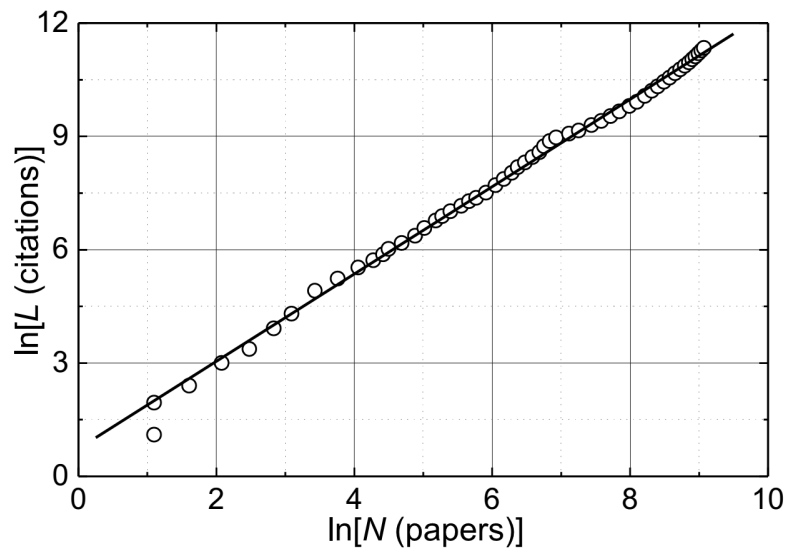

Fig. 5. Relationship between $\ln L(t)$ on $\ln N(t)$ according to Eq. (13). Linear plot is drawn with best-fit parameters listed in Table $\mathrm{V}$.

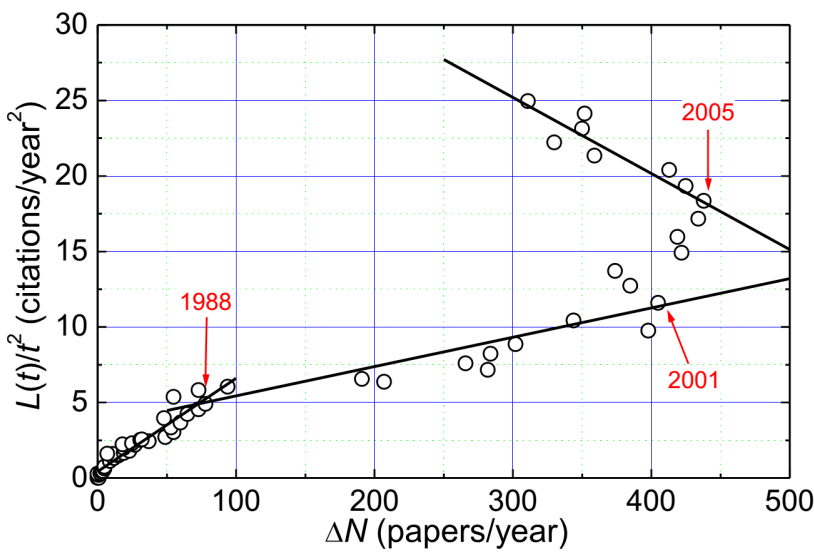

Fig. 6. Plots of $L(t) / t^{2}$ against $\Delta N(t)$ according to Eq. (14). Linear plots are drawn according to best-fit parameters listed in Table IV.

Figure 5 shows the plot of $\ln L(t)$ against $\ln N(t)$ according to Eq. (13) where the linear plot is drawn with the best-fit parameters: slope $=1.1557 \pm 0.0072$ and intercept $\ln \left(\lambda_{0} t / \Theta_{0}\right)=0.733 \pm 0.048$, i.e. $\lambda_{0} t / \Theta_{0}=$ $2.08 \pm 0.10$. As predicted by Eq. (13), the value of the slope is close to unity but a constant value of $\ln \left(\lambda_{0} t / \Theta_{0}\right)$ is expected when $\Theta_{0}$ increases linearly with $t$ and the citability parameter $\lambda_{0}$ remains constant. In fact, it was reported that $\Theta_{0}$ increases linearly with $t$ [19].

From an analysis of the time dependence of the scientific activities of different authors determined by the cumulative number $L(t)$ of citations of their papers, the present author [19] found that the time dependence of $\mathrm{cu}-$ mulative number $L(t)$ of citations of papers reveals one or more citation periods during the publication careers of different authors. In order to analyze and compare the scientific activities of different authors, the author suggested to use the parameter $L(t) / t^{2}$, defined as citation acceleration $a$. Considering $L / t^{2}$ as a time-dependent variable related to the time-dependence of $\Delta N(t)$, from Eq. (12) the relationship between $\left[L(t) / t^{2}\right]$ and $\Delta N(t)$ in the time interval between $t_{0}$ and $t$ may be written as

$$
\frac{L(t)}{t^{2}}=\frac{L\left(t_{0}\right)}{t_{0}^{2}}+\frac{\lambda_{0}}{\Theta_{0}}\left[\Delta N(t)-\Delta N\left(t_{0}\right)\right],
$$

where we have taken $q_{0}=2$ and the term $\left[L\left(t_{0}\right) / t_{0}^{2}\right]$ corresponds to the paper publication rate $\Delta N\left(t_{0}\right)$ at the reference time $t_{0}$. Note that the proportionality term $\lambda_{0} / \Theta_{0}>0$, and $\Delta N\left(t_{0}\right)=0$ at $t_{0}=0$ but for all values of $t_{0}>0$ when $\Delta N\left(t_{0}\right)>0,\left[L\left(t_{0}\right) / t_{0}^{2}\right]>0$. Similarly, the term $\left[L(t) / t^{2}\right]>0$ for all values of $t$. However, depending on the $\Delta N(t)$ interval, the slope $\lambda_{0} / \Theta_{0}$ can be positive as well as negative. When $\left[\Delta N(t)-\Delta N\left(t_{0}\right)\right]>0$, the slope $\lambda_{0} / \Theta_{0}>0$; but when $\left[\Delta N(t)-\Delta N\left(t_{0}\right)\right]<0, \lambda_{0} / \Theta_{0}<0$.

Figure 6 presents the plots of $L(t) / t^{2}$ against $\Delta N(t)$ according to Eq. (14). As seen from the figure, the data follow three different linear parts: (1) $0<\Delta N(t)<80$ papers/year corresponding to $0<\left[L(t) / t^{2}\right]<5$ citations $/$ year $^{2}$ covering the publication output period up to $Y \approx 1988,(2) 80<\Delta N(t)<405$ papers/year corresponding to $5<\left[L(t) / t^{2}\right]<14$ citations $/$ year $^{2}$ covering the period $1988<Y<2001$, and $(3) 440>\Delta N(t)>300$ papers/year corresponding to $17<\left[L(t) / t^{2}\right]<25$ citations/year ${ }^{2}$ for $Y>2005$. The best-fit values of the intercept $a_{0}$ and the slope $b$ in the three $\Delta N(t)$ intervals are listed in Table $\mathrm{V}$, where

$$
a_{0}=\left[\frac{L\left(t_{0}\right)}{t_{0}^{2}}-\frac{\lambda_{0}}{\Theta_{0}} \Delta N\left(t_{0}\right)\right], \quad b=\frac{\lambda_{0}}{\Theta_{0}} .
$$

Note that the slope $b>0$ in the first two intervals, whereas $b<0$ in the last interval. These slopes are directly connected with the nature of the plots of $\Delta N(t)$ against publication year $Y$ seen in Fig. 3a. The slope $b>$ 0 in the first two intervals because $\left[\Delta N(t)-\Delta N\left(t_{0}\right)\right]>0$ in these intervals, but $b<0$ in the last interval because $\left[\Delta N(t)-\Delta N\left(t_{0}\right)\right]<0$

\section{Summary and conclusions}

As found in previous papers [8, 9, 16, 18, 19], it is shown that the basic concepts of progressive nucleation mechanism originally proposed for crystal growth can satisfactorily be applied to explain the citation behavior of papers published on the Czochralski method of crystal growth. Application of mathematical equations based on 
progressive nucleation mechanism to explain the growth dynamics of papers related to the Czochralski method of crystal growth and citations to these papers published in the scientific literature revealed that: (1) cumulative papers $N(t)$ and cumulative citations $L(t)$ are mutually related, and (2) the citation behavior of papers published on the Czochralski method follows three distinct periods: 1954-1988, 1988-2001 and 2001-2012, which are related to the nature of the plots of $\Delta N(t)$ against publication time $t$.

\section{References}

[1] G. Teal, J.B. Little, Phys. Rev. 78, 647 (1950).

[2] W.K. Burton, N. Cabrera, F.C. Frank, Philos. Trans. R. Soc. London A 243, 299 (1951).

[3] H.E. Buckley, Crystal Growth, Wiley, New York 1951.

[4] K.-Th. Wilke, J. Bohm, Kristallzüchtung (Crystal Growth), Deutscher Verlag der Wissenschaften, Berlin 1988, p. 679, (in German).

[5] P.E. Tomaszewski, "Professor Jan Czochralski (18851953) and his contribution to the art and science of crystal growth", AACG Newsletter 27, 12 (1998).

[6] P.E. Tomaszewski, Return: About Jan Czochralski, ATUT, Wrocław 2012 (in Polish).
[7] J. Żmija, Technology of Obtaining Single Crystals, WSI, Radom 1981, p. 91 (in Polish); Obtaining Single Crystals, PWN, Warszawa 1988, p. 91 (in Polish).

[8] K. Sangwal, J. Informetr. 5, 554 (2011).

[9] K. Sangwal, Scientometrics 92, 575 (2012).

[10] D.J. De Solla Price, Little Science, Big Science, Columbia University Press, New York 1963.

[11] L. Egghe, I.K. Ravichandra Rao, Scientometrics 25, 5 (1992).

[12] B.M. Gupta, L. Sharma, C.R. Karisiddappa, Scientometrics 33, 187 (1995).

[13] B.M. Gupta, S. Kumar, S.L. Sangam, C.R Karisiddappa, Scientometrics 53, 161 (2002).

[14] I.K. Ravichandra Rao, D. Srivastava, J. Informetr. 4, 249 (2010).

[15] C.-Y. Wong, K.-L. Goh, J. Informetr. 4, 460 (2010).

[16] K. Sangwal, Scientometrics 92, 643 (2012).

[17] D. Kashchiev, Nucleation: Basic Theory with Applications, Butterworth-Heinemann, Oxford 2000.

[18] K. Sangwal, J. Informetr. 5, 529 (2011).

[19] K. Sangwal, Inform. Proc. Manag. 49, 757 (2013). 